\title{
The role of epigenetic factors in descendants from Assisted Reproductive Techniques (ART)
}

\author{
Vasilios Pergialiotis' ${ }^{1}$ Anastasia Prodromidou' ${ }^{1}$ Evangelia Dimitroulia ${ }^{2}$, Dimitrios Loutradis ${ }^{3}$ \\ ${ }^{1} 1^{\text {st }}$ Department of Obstetrics \& Gynecology, Medical School of Athens, National and Kapodistrian University \\ of Athens, "Alexandra" Hospital, Athens, Greece \\ 2Department of Microbiology, Medical School of Athens, National and Kapodistrian University of Athens, Athens, \\ Greece \\ ${ }^{3}$ Medical School of Athens, National and Kapodistrian University of Athens, Athens, Greece
}

\section{Corresponding Author}

Anastasia Prodromidou, MD, MSc, PhD, $1^{\text {st }}$ Department of Obstetrics \& Gynecology, Medical School, National and Kapodistrian University of Athens, "Alexandra" Hospital, Athens, Greece. e-mail: a.prodromidou@hotmail.com

\begin{abstract}
Altogether, Assisted Reproductive Techniques (ART) are likely to cause some epigenetic changes in the offspring, which might consist the molecular basis of complex characteristics and diseases. The present review contributes a large biochemical dataset of a well-defined group of pre- pubertal in vitro fertilization (IVF) and intracytoplasmic sperm injection (ICSI) conceived children in order to detect the potential effect in the offspring's health. Additionally, the relevant usefulness of metabolomics and proteomics are also investigated. The outcomes indicate early insulin resistance in ICSI-offspring which can set the basis for further research in the field so as to identify the respective pathophysiological pathways and mechanisms of action. The data support that metabolomics may unravel metabolic variances before they become clinically or biochemically evident, underlining its utility in the ART research.
\end{abstract}

Key words: Epigenetics, IVF, ICSI, offspring's, proteomics, metabolomics

\section{Introduction}

Since the birth of Louise Brown in 1978, a rapid development in assisted reproductive techniques (ART) has been observed. Assisted reproduction techniques, including in vitro fertilization (IVF) and intracytoplasmic sperm injection (ICSI), have been widely used for infertility. The application of them has been proved beneficial for millions of couples who suffer from infertility. The safety of the applied techniques has gained significant attention as the number of offsprings from ART has increased during the past decades. Whether children that were born through assisted reproduction are more likely to develop health problems than those conceived spontaneous is a matter of controversy. 
The regimens that are administered for ovarian stimulation, the in vitro culture of the embryos, cryopreservation, and the use of potentially structurally and genetically abnormal sperm in ICSI are considered independent risk factors. Additionally, multiple pregnancies as due to transfer of more than one embryos have been associated with higher rates of prematurity and low birth weight.

The objective of the present study was to review the potential epigenetic factors that can affect the offspring of ART.

\section{Main outcomes}

A total of 6 studies that investigate the characteristics of children born through assisted reproduction, were carried out by the collaboration of the $1^{\text {st }} \mathrm{De}$ partment of Obstetrics and Gynecology of Alexandra Hospital and the $1^{\text {st }}$ Department of Pediatrics of the University of Athen ${ }^{1-6}$. The initial conceptualization and implementation of the project was initiated by Professor G. Chroussos in collaboration with Professor Ch. Kanaka-Gantenbein, from the $1^{\text {st }}$ Pediatric Clinic of National and Kapodistrian University of Athens, "Agia Sofia" Hospital.

With regards to the $1^{\text {st }}$ study, the potential epigenetic effects of ART techniques were examined. In particular, the thyroid function of 106 children born from IVF (study group) was compared with 68 controls that have been natural conceived(control) ${ }^{1}$. The age of the included subjects ranged from 4 to 14 years. The evaluated parameters in all children were T3, T4, TSH and antithyroid peroxidase and antithyroglobulin antibodies titers. A significant increase in serum TSH was observed in children from IVF group compared with controls along with a mild resistance of TSH in the thyroid gland. This was not associated with respective alterations in antithyroid antibody levels. The aforementioned outcomes indicate a potential involvement of epigenetic developmental abnormalities that may be associated with the pre- implantation manipulations on the fetus.

Another study found that offspring from conventional IVF showed significantly higher systolic and diastolic blood pressure, as well as triglyceride levels when compared to the matched control-spontaneous conception group ${ }^{2}$. The differences remained significant even after correction of the potential confounding factors including birth weight and multiple pregnancies ${ }^{2}$. No difference was observed in insulin resistance, in glucose to insulin ratio, as well as in inflammatory response parameters ${ }^{2}$.

Three years later the same study group evaluated the role of some other metabolic values in IVF children including lipokalins, such as retinolbinding protein 4 (RBP-4) and neutrophil gelatinaseassociated lipocalin (NGAL), as well as visfatin and the respective role of gender ${ }^{3}$. The aforementioned factors are associated with glucose intolerance and could help in the early diagnosis of potential metabolic disorders. More specifically, 100 IVF children aged between 4 and 14 years were compared with 60 controls that were born after spontaneous conception $^{3}$. The outcomes indicated that IVF children presented significantly elevated levels of both RBP-4 ( $p=0.009)$ and NGAL ( $p=0.028)$ compared to controls $^{3}$. Furthermore, females born from IVF presented higher RBP-4 values ( $p=0.002)$ while NGAL values were greater in males born after IVF ( $\mathrm{p}=0.021)$ when compared to controls ${ }^{3}$, suggesting early metabolic derangements that could be attributed to an epigenetic phenomenon (Figure 1).

The study by Gkourogianni et al examined the potential changes in the metabolic profile and insulin resistance in 42 pre-pubertal children that were born after ICSI and compared them with 42 age-matched controls who were born after natural conception ${ }^{4}$. Metabolic analysis of ICSI children revealed lower urea and levels of YKL- 40 and hsCRP inflammatory and cardiometabolic markers along with elevated triiodothyronine levels when compared to controls ${ }^{4}$. 

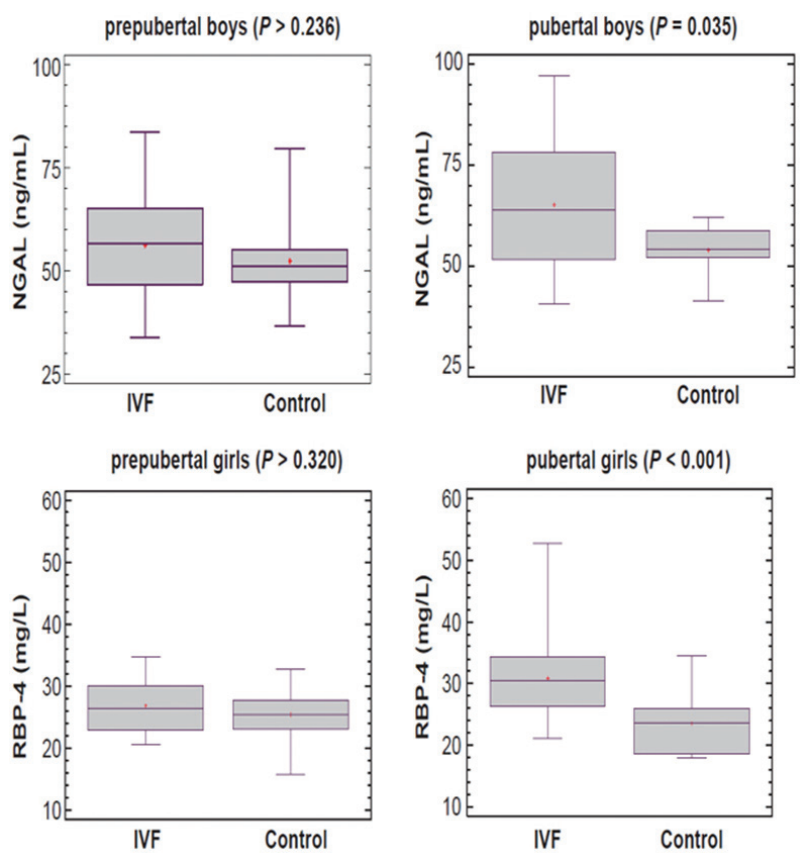

Figure 1. Comparison of neutrophil geltinase-associated lipocalin (NGAL) in prepudertal and pubertal boys and of retinol-bonding protein 4 (RBP-4) and pubertal girls between in vitro fertilization children and controls ${ }^{3}$.

Hyperthyroidism can lead to glucose intolerance and insulin resistance ${ }^{7}$. An additional analysis was performed in a subgroup of 10 girls from each group (ICSI and natural conception matched for age, gestational age, birth weight and singleton or twin pregnancy) with the use of Gas Chromatography-Mass Spectometry (GC-MS) for metabolic analysis in blood plasma ${ }^{4}$. The analysis showed significant differences in 37 out of 70 metabolites among ICSI girls and natural conceived controls ${ }^{4}$. The vast majority of them were associated with metabolic syndrome, insulin resistance and obesity. The outcomes of this study raise high suspicion for a significant predisposition of ICSI children to cardiometabolic diseased in their adulthood and highlights the important role of metabolomics in the research of epigenetic effects of ART which can predict metabolic alterations before their biochemical or clinical onset (Figure 2).
In 2017 the study by Kosteria et al examined the outcomes of the proteomic analysis of the aforementioned population (ICSI children versus natural conception controls, matched for age and sex $)^{5}$. They assessed the potential changes in plasma proteomic profile among 10 individual couples of children from the two groups searching for potential cardiometabolic markers. To counteract factors that can potentially influence the metabolic status, the couples of children were matched for sex, age, number of embryos and birth weight while pubertal children were not included. When patients' characteristics were compared, ICSI group was found with decreased gestational age at delivery, birth weight, increase in the number of caesarean sections and maternal age. However, this was not associated with adverse perinatal outcomes. With regards to the proteomic analysis, of the 22 proteins that were found to be expressed differentially, 19 over- and 3 under-expressed proteins in the ICSI group were identified in the ICSI group. The majority of the overexpressed proteins are involved in acute phase reactions, blood coagulation, and activation of iron and lipid metabolism pathways, indicating an adverse subclinical cardiometabolic profile (Figure 3).

More recently, a matched for race, age and sex cohort study evaluated the blood lipid profile and more specifically proprotein convertase subtilisin / kexin type 9 (PCSK9) and serum lipoprotein (a) (Lp(a)) levels in 73 children born from ART (either ICSI- $n=33$ or classic IVF- $n=40$ ) and in 73 children born after natural conception ${ }^{6}$. PCSK9 and Lp [a] levels are known factors that have been associated with cardiovascular risk ${ }^{8}$. Classification according to age ( $<8$ years, $8-10$ years, $\geq 10$ years) was used for the comparison among the groups. The outcomes of the study indicate that in the total patient cohort, PCSK9 levels were strongly related to total cholesterol, lowdensity lipoprotein, and systolic blood pressure, as proved by univariate analysis. In addition to this, 


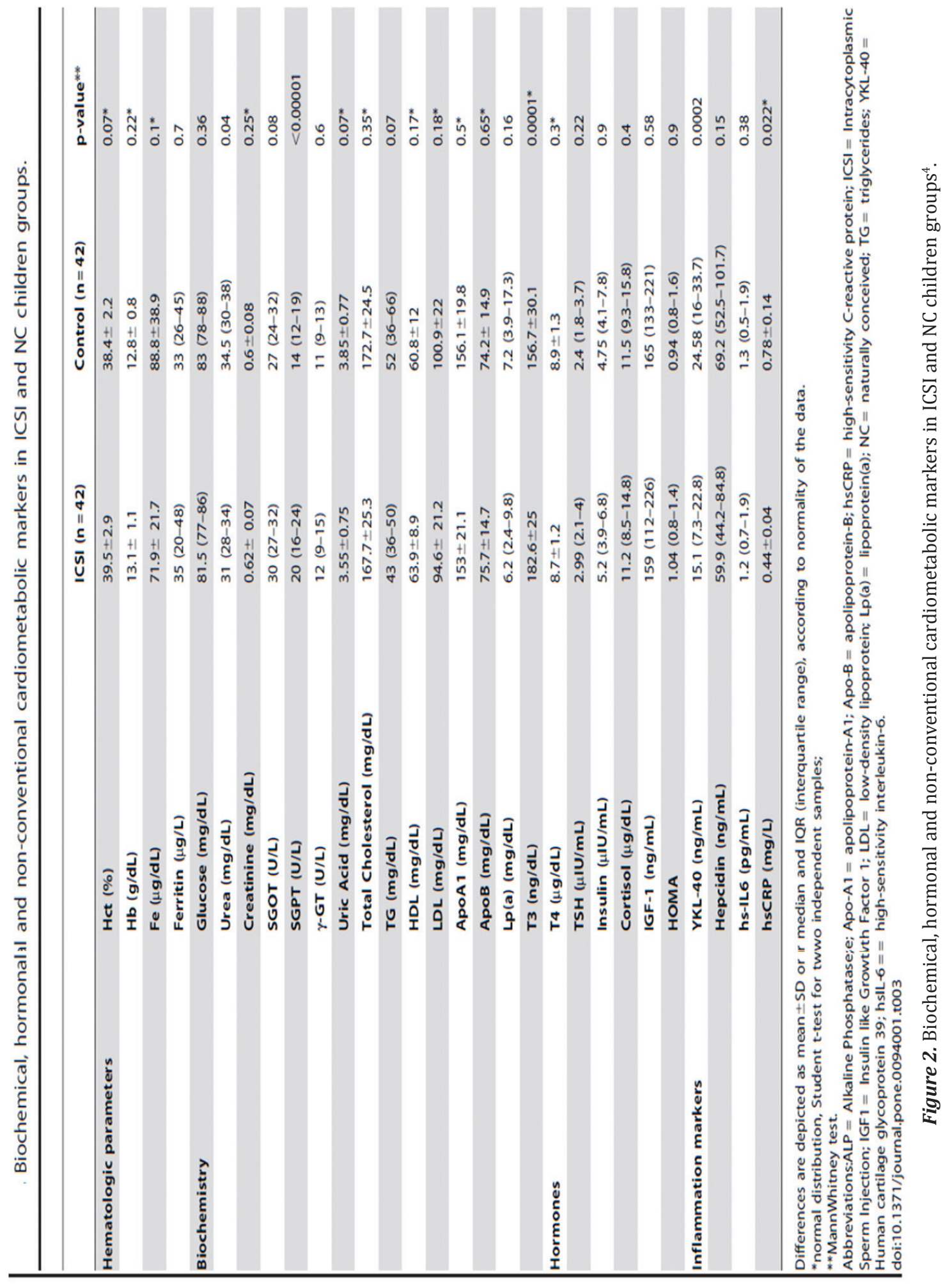




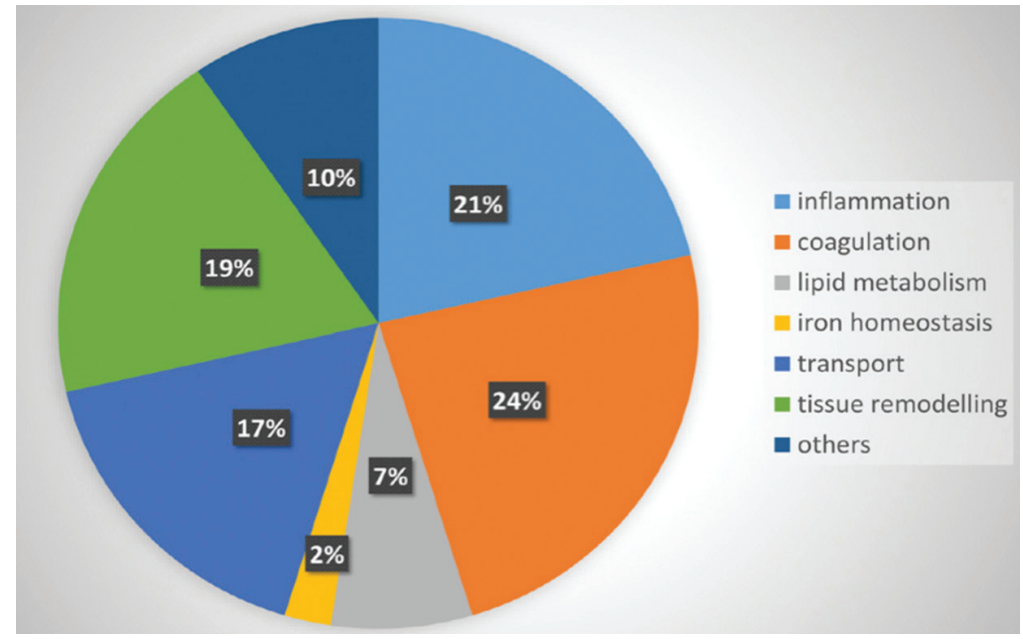

Figure 3. Representation of functional classification of identified, differentially expressed proteins (\% total number of identified proteins). Proteins were classified according to their major funtions according to the uniprot database ${ }^{5}$.

Lp (a) levels were increased with age, birth weight, height, waist-to-ratio hip, high-sensitivity C-reactive protein and apolipoprotein-B, insulin resistance and insulin. The comparison of lipid biomarkers among ART and natural conception children did not reveal any difference. On the other hand, a correlation was detected among age groups and conception method, indicating that in ART children PCSK9 levels were increased with age, while in natural conceived ones, PCSK9 were decreased with age. Children born from

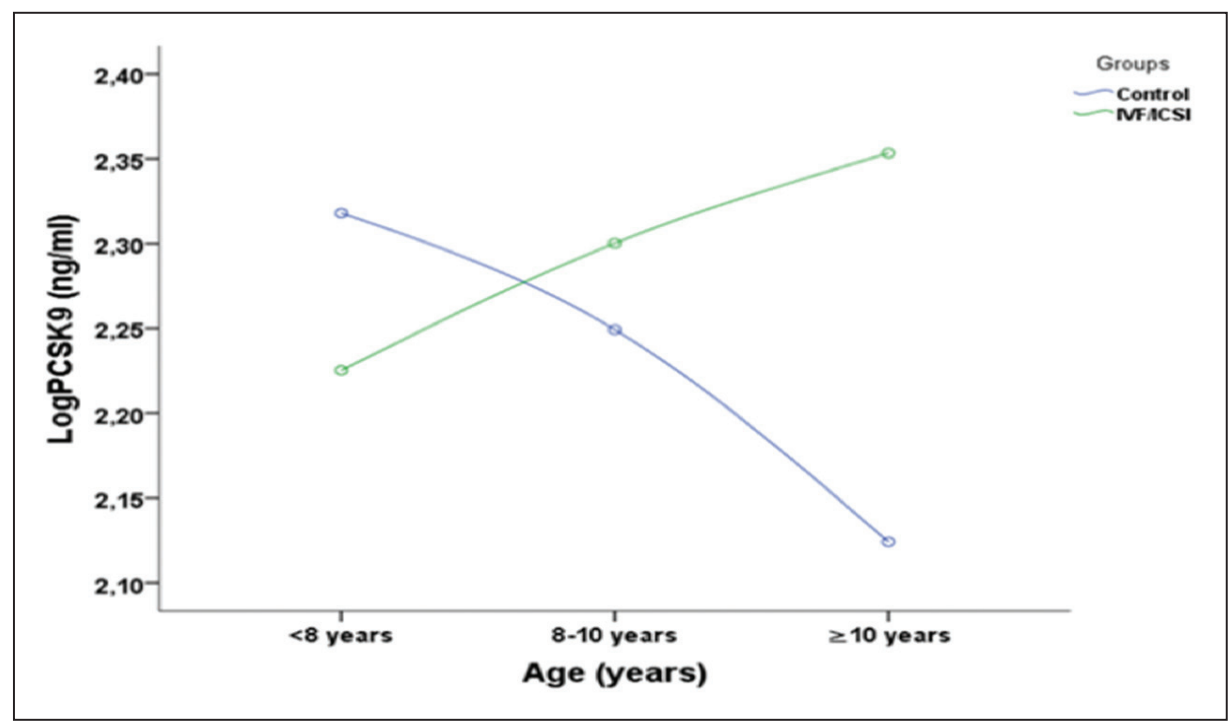

Figure 4. PCSK9 levels increase with age in ART children, indicating a gradual deterioration of lipidemic profile that could lead to increased cardiovascular risk. Moreover, our results indicate that ART method may be of importance given that classic IVFis associated with higher levels of $L p(a)^{6}$. 
IVF showed significantly elevated mean levels of adjusted Lp (a) when compared to both ICSI (13.5 vs. $6.8 \mathrm{mg} / \mathrm{dl}, \mathrm{p}=0.010)$ and controls (12.3 vs. 8.3 $\mathrm{mg} / \mathrm{dl}, \mathrm{p}=0.048$ ) (Figure 4).

The increase in PCSK9 levels that was observed with age in ART children, could be suggestive of a gradual worsening of the lipidemic profile that could increase the risk for cardiovascular events. In addition, the outcomes of the study showed that the ART method may be relevant, based on the higher levels of Lp (a) in the analysis of IVF children.

ART is likely to cause some epigenetic changes in the offspring, which might be the molecular basis of complex traits and diseases. However, it is still unclear whether the small differences observed in several studies represent a real difference between ART-conceived and NC children. Larger studies with long-term follow-up are needed to fully answer these questions

\section{Acknowledgements}

None

\section{References}

1. Sakka SD, Malamitsi-Puchner A, Loutradis D, Chrousos GP, Kanaka-Gantenbein C. Euthyroid hyperthyrotropinemia in children born after in vitro fertilization. The Journal of clinical endocrinology and metabolism 2009;94(4):1338-41 https:// pubmed.ncbi.nlm.nih.gov/19190111/.

2. Sakka SD, Loutradis D, Kanaka-Gantenbein C et al. Absence of insulin resistance and low-grade inflammation despite early metabolic syndrome manifestations in children born after in vitro fertilization. Fertil Steril 2010;94(5):1693-9 https:// pubmed.ncbi.nlm.nih.gov/20045517/.

3. Sakka SD, Margeli A, Loutradis D, Chrousos GP, Pa- passotiriou I, Kanaka-Gantenbein C. Gender dimorphic increase in RBP-4 and NGAL in children born after IVF: an epigenetic phenomenon? European journal of clinical investigation 2013;43(5):43948 https://pubmed.ncbi.nlm.nih.gov/23496280/.

4. Gkourogianni A, Kosteria I, Telonis AG et al. Plasma metabolomic profiling suggests early indications for predisposition to latent insulin resistance in children conceived by ICSI. PloS one 2014;9(4):e94001 https://pubmed.ncbi.nlm.nih. gov/24728198/.

5. Kosteria I, Tsangaris GT, Gkourogianni A et al. Proteomics of Children Born After Intracytoplasmic Sperm Injection Reveal Indices of an Adverse Cardiometabolic Profile. Journal of the Endocrine Society 2017;1(4):288-301 https://pubmed.ncbi. nlm.nih.gov/29264487/.

6. Vlachopoulos C, Kosteria I, Sakka S et al. PCSK9 and $\mathrm{Lp}(\mathrm{a})$ levels of children born after assisted reproduction technologies. Journal of assisted reproduction and genetics 2019;36(6):1091-9 https:// pubmed.ncbi.nlm.nih.gov/31079266/.

7. Brenta G. Why can insulin resistance be a natural consequence of thyroid dysfunction? Journal of thyroid research 2011;2011:152850 https:// pubmed.ncbi.nlm.nih.gov/21941681/.

8. Vlachopoulos C, Terentes-Printzios D, Georgiopoulos $\mathrm{G}$ et al. Prediction of cardiovascular events with levels of proprotein convertase subtilisin/kexin type 9: A systematic review and meta-analysis. Atherosclerosis 2016;252:50-60 https://pubmed. ncbi.nlm.nih.gov/27501130/.

Received 05-11-20

Revised 10-11-20

Accepted 16-11-20 